\title{
Detection of CCNE1/URI (19q12) amplification by in situ hybridisation is common in high grade and type II endometrial cancer
}

\author{
Aurelia Noske ${ }^{1}$, Simone Brandt ${ }^{1}$, Nadejda Valtcheva ${ }^{1}$, Ulrich Wagner ${ }^{1}$, Qing Zhong ${ }^{1}$, \\ Elisa Bellini ${ }^{1}$, Daniel Fink ${ }^{2}$, Ellen C. Obermann ${ }^{3}$, Holger Moch ${ }^{1}$, Peter J. Wild ${ }^{1}$ \\ ${ }^{1}$ Institute of Surgical Pathology, University Hospital Zurich, Zurich, Switzerland \\ ${ }^{2}$ Department of Gynaecology, University Hospital Zurich, Zurich, Switzerland \\ ${ }^{3}$ Institute of Pathology, University Hospital Basel, Basel, Switzerland \\ Correspondence to: Aurelia Noske, email: aurelia.noske@tum.de
}

Keywords: endometrial cancer, in situ hybridisation, 19q12

Received: May 17, $2016 \quad$ Accepted: August 13, 2016

Published: August 25, 2016

\section{ABSTRACT}

One TCGA subgroup of endometrial cancer (EC) is characterised by extensive genomic DNA copy number alterations. CCNE1 located at $19 q 12$ is frequently amplified in EC and a target for anti-cancer therapy. The relevance of URI, also located at 19q12, is unknown. To evaluate the prevalence of $19 q 12$ (CCNE1/URI) in EC, we investigated different histologic types by in situ hybridisation (ISH) and copy number assay. We applied a previously established 19q12 ISH for the detection of CCNE1/URI copy numbers in EC $(n=270)$ using conventional bright field microscopy. In a subset $(n=21)$, 19q12 amplification status was validated by OncoScan assay. Manual ISH was controlled by a recently developed computational ISHProfiler algorithm. Associations of 19q12 status with Cyclin E1, URI and p53 expression, and clinico-pathological parameters were tested.

Amplification of 19q12 (CCNE1/URI) was found in $10.4 \%(28 / 270)$ and was significantly associated with type II EC (high grade and non-endometrioid; $p<0.0001$ ), advanced FIGO stage $(p=0.001)$, high Cyclin E1 expression $(p=0.008)$ and aberrant p53 expression $(p=0.04)$. 19q12 ISH data were confirmed by OncoScan and computational ISHProfiler techniques. The $19 q 12$ in situ hybridisation is a feasible and robust biomarker assay in molecular pathology. Amplification of CCNE1/URI predominantly occurred in type II endometrial cancer. Prospective clinical trials are warranted to assess the utility of combined 19q12 amplification and Cyclin E1/URI protein expression analysis for the prediction of therapeutic response to chemotherapy and/or cyclin-dependent kinase inhibitors in patients with endometrial cancer.

\section{INTRODUCTION}

Endometrial cancer (EC) is traditionally subdivided into types I and II based on histological type, tumour differentiation and clinical features [1]. Type I includes low-grade endometrioid EC and variants, showing a favourable prognosis. Conversely, type II tumours comprise high-grade and non-endometrioid cancers (serous and clear cell) that are less frequent (10-20\%) but more aggressive with characteristic poor outcomes. At molecular levels, types I and II EC share several genetic abnormalities (frequent PTEN, PIK3CA and KRAS mutations). Contrary to type I, most type II carcinomas exhibit a high frequency of TP53 mutations. The Cancer Genome Atlas (TCGA) analysis of endometrial carcinomas revealed four genomic groups; among them was the 'copy number $(\mathrm{CN})$ high' group termed 'serous-like', comprising serous, mixed and high-grade endometrioid ECs showing frequent TP53 mutations, CCNE1 (19q12) amplification, rare microsatellite instability and fewer PTEN mutations than other ECs [2].

Genomic amplification of CCNE1 and increased expression of the encoded protein Cyclin E1 has been previously shown in serous EC [3, 4]. CCNE1 drives the 
genesis of uterine serous carcinomas mainly by activating cell-cycle progression through $\mathrm{CDK} 2$ activation, $\mathrm{Rb}$ phosphorylation and E2F-1-mediated transcription [3]. However, the 19q12 amplicon comprises several genes along with CCNE1, including URI (C19Orf2, RPB5mediating protein), C19Orf12, POP4 and PLEKHF1. We and others recently observed $U R I$ amplification in carcinomas of the ovary [5-7] and endometrium [6]. The URI protein belongs to the prefoldin family of molecular chaperones involved in translational control-related pathways [8]. URI overexpression in ovarian cancer cells promotes cell survival and contributes to the oncogenic effect of 19q12 amplification [6].

Patients with type II EC have a high relapse risk and poor prognosis, particularly those belonging to the 'copy number high' EC genomic subgroup [2]. Guidelines recommend treatment by surgery, adjuvant radiation and chemotherapy in patients with high-grade and/or advanced EC. Currently, the long-term effectiveness of chemotherapy (usually platin-taxane-based) is uncertain [9]. Although therapy results in an initial complete response, resistance development is a major problem [10]. Chemoresistance in high-grade serous ovarian carcinomas is attributed to amplified CCNE1 [11, 12]. Since endometrial and ovarian carcinomas show a genomic relationship, the $19 q 12$ amplification status may also have an impact on EC therapy. Additionally, while several targeted drug clinical trials were completed, no targeted therapies were approved for EC. Novel drugs and combinations are constantly being introduced, but there are few predictive markers and tests for patient selection. The 19q12 amplicon is a potential predictive marker for response to conventional chemotherapy and CDK inhibitors [12-14]. Treatment decisions could potentially depend on the $19 \mathrm{q} 12(C C N E 1 / U R I)$ copy number status in combination with Cyclin E1 and/or URI protein expression.

We evaluated the prevalence and degree of $19 \mathrm{q} 12$ (including CCNE1 and URI) amplification in a large group of archived EC samples using a recently established chromogenic in situ hybridisation (ISH) assay for automated 19q12 detection with immunohistochemical Cyclin E1 and URI protein expression [7]. To validate the 19q12 ISH data, we analysed a subset of samples for $\mathrm{CN}$ changes using the Affymetrix OncoScan assay. Additionally, manually assessed 19q12 ISH was independently scored by a computational ISHProfiler algorithm.

\section{RESULTS}

\section{EC sample characteristics}

Totally, 436 endometrial carcinomas were studied. Endometrioid carcinoma (and variants) was the most common subtype $(361,83.8 \%)$. Less frequent was the non-endometrioid subtype $(61,12.6 \%)$. In 16 samples (3.6\%), the histological type could not be determined. According to the traditional histological EC categorisation, $310(71.1 \%)$ were type I (low-grade endometrioid and mucinous) and 102 were type II (high-grade endometrioid and non-endometrioid). Subtyping was impossible in 5.5\% samples. Most cases were diagnosed in an early FIGO stage $(58.9 \%)$. Characteristics are listed in Table 1 .

\section{9q12 amplification occurs more frequently in type II EC}

The 19q12 ISH assay was applied to two TMA cohorts (Basel and Zurich-TMA), both including different histological EC subtypes. Tissue cores containing at least 50 tumour cells were evaluated to calculate $\mathrm{CN}$. Due to tumour tissue paucity, crush artefacts or weak ISH signals, not all tissue cores were amenable to analysis. Assay interpretation was possible for 270 tissue samples and was performed by a board certified pathologist (A.N.). 19 q12 enumeration and chromosome 19 signals were manually completed for 50 tumour nuclei/sample with amplification defined as $19 \mathrm{q} 12 / \mathrm{chr} 19$ ratio $\geq 2.0[7,15]$. Using this cut-off, copy number alterations were found in $28 / 270$ carcinomas (10.4\%). 19q12 amplification was significantly associated with type II EC (comprising highgrade and non-endometrioid cancer) and advanced FIGO stage (Table 2, Figure 1A-1B). In univariate Kaplan-Meier analysis, patients with 19q12 amplified carcinomas tended to have worse overall survival, but this trend did not reach statistical significance (log-rank test, $p=0.075$ ).

\section{Cyclin E1 and URI protein expression}

Cyclin E1 immunohistochemical analysis was possible in 412 carcinomas. The $\mathrm{H}$-score (range 0-300) and afterwards the median H-score (140) was calculated to distinguish low from high Cyclin E1 expression. High expression levels were observed in 208/412 cases (50.5\%). Increased Cyclin E1 expression was significantly more frequent in type II and non-endometrioid EC (Table 2, Figure 1C). In univariate Kaplan-Meier analysis and univariate Cox regression analysis, patients with high Cyclin E1 expressing carcinomas had significantly shorter overall survival time than patients with low Cyclin E1 expression (log-rank test, $p=0.017$; $\mathrm{HR}=1.59,95 \% \mathrm{CI}$, 1.09-2.3). However, an independent prognostic value of Cyclin E1 overexpression was not confirmed by multivariate COX regression analysis adjusted for other parameters (patient age, tumour subtype and FIGO stage; $p=0.079)$.

Evaluation of URI protein expression by immunohistochemistry was possible in 416 carcinomas. URI expression was exclusively detected in cytoplasm. For statistics, we categorised the expression as recently described [7]. Moderate and strong cytoplasmic staining 
Table 1: Pathological characteristics of EC samples $(n=436)$

\begin{tabular}{lc}
\hline Characteristics & $\mathbf{n}(\mathbf{\%})$ \\
\hline Histological type & $359(83.3)$ \\
endometrioid & $2(0.5)$ \\
mucinous & $22(5.1)$ \\
serous & $17(3.9)$ \\
clear cell & $12(2.8)$ \\
carcinosarcoma (MMMT) & $8(1.9)$ \\
undifferentiated & $16(3.6)$ \\
unclassifiable & \\
Type I \& II category & $310(71.1)$ \\
Type I & $102(23.4)$ \\
Type II & $24(5.5)$ \\
unclassifiable & \\
FIGO stage & $257(58.9)$ \\
early & $84(19.3)$ \\
late & $95(21.8)$ \\
missing &
\end{tabular}

MMMT: malignant mixed Müllerian tumour

Table 2: Associations between pathological parameters and 19q12 amplification, Cyclin E1, and URI protein expression

\begin{tabular}{|c|c|c|c|c|c|c|}
\hline & $\begin{array}{c}\text { 19q12 } \\
\text { Amplification } \\
\text { n (\%) }\end{array}$ & p-value* & $\begin{array}{c}\text { Cyclin E1 high } \\
\text { n (\%) }\end{array}$ & p-value* & $\begin{array}{c}\text { URI high } \\
\text { n (\%) }\end{array}$ & p-value* \\
\hline Histology ${ }^{1}$ & & $<0.0001$ & & $<0.0001$ & & 0.009 \\
\hline endometrioid & $13 / 230(5.6)$ & & $162 / 350(46.2)$ & & $38 / 353(10.7)$ & \\
\hline $\begin{array}{l}\text { non- } \\
\text { endometrioid }\end{array}$ & $15 / 37(40.5)$ & & $44 / 58(75.8)$ & & $14 / 58(24.1)$ & \\
\hline Tumour type ${ }^{2}$ & & $<0.0001$ & & $<0.001$ & & 0.010 \\
\hline type I & $8 / 189(4.2)$ & & $137 / 300(45.6)$ & & $31 / 302(10.2)$ & \\
\hline type II & $19 / 70(27.1)$ & & $65 / 100(65)$ & & 21/101 (20.8) & \\
\hline FIGO stage & & 0.002 & & 0.31 & & 0.83 \\
\hline early & $8 / 148(5.4)$ & & $126 / 244(51.6)$ & & $24 / 248(9.6)$ & \\
\hline late & $11 / 51(21.5)$ & & $49 / 84(58.3)$ & & $7 / 84(8.3)$ & \\
\hline
\end{tabular}

* Fisher's Exact Test

${ }^{1}$ non-endometrioid carcinomas (including serous, clear cell, undifferentiated, carcinosarcoma)

${ }^{2}$ type I endometrioid (low grade, G1-2) and mucinous; type II endometrioid (high grade, G3) and non-endometrioid histology 

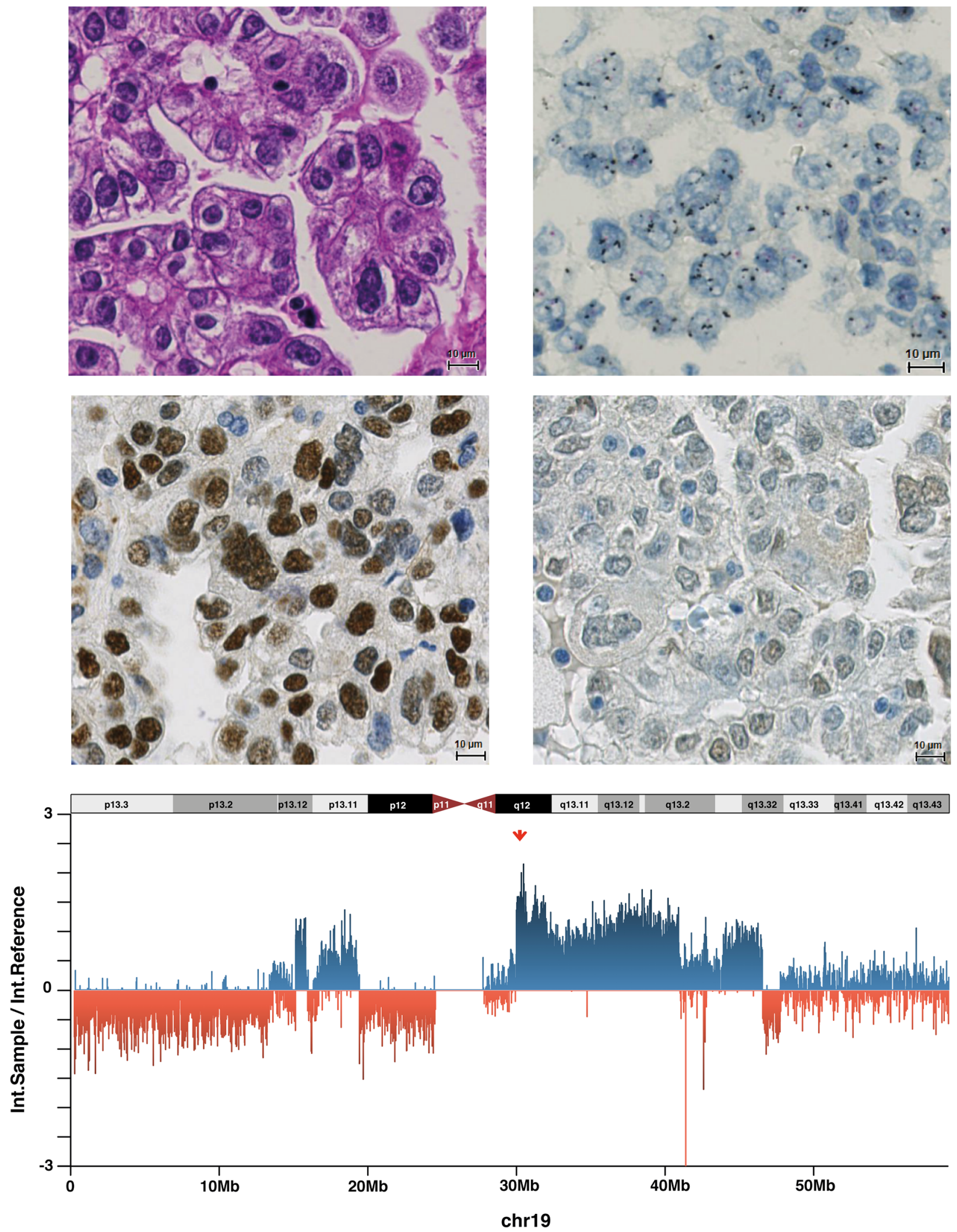

Figure 1: Endometrial cancer (Case 33) with 19q12 amplification, high Cyclin E1 expression, URI negativity and high 19q12 copy number. A. Endometrial carcinoma of the clear cell type belonging to type II (HE staining). B. Amplification of 19q12 in a dual-colour ISH (19q12 black signals, reference red signals). C. High Cyclin E1 expression in the tumour cell nuclei. D. Absence of URI expression. E. Intensity ratios (intensity sample/intensity reference) for all chromosome 19-related probes of the OncoScan assay. High copy numbers at 19q12 (CCNE1/URI) locus are indicated by the red arrow. 
(defined as URI-positive) was observed in $12.5 \%$ of the carcinomas (52/416) but neither associated with pathological features (Table 2, Figure 1D) nor overall survival (log-rank test, $p=0.68$ ).

\section{Association of 19q12 amplification status with CCNE1 and URI expression}

As the $19 \mathrm{q} 12$ ISH assay covers both CCNE1 and $U R I$, we tested the associations of $19 \mathrm{q} 12$ amplification status with Cyclin E1 and URI protein expression levels (Table 3). 19q12 amplification was significantly associated with high Cyclin E1 expression ( $p=0.008$, Fisher's exact test). However, there were cases with high Cyclin E1 expression without 19q12 amplification and 19q12amplified tumours with low Cyclin E1 expression. No association between 19q12 amplification status and URI expression was found ( $p=0.593$, Fisher's exact test). Cyclin E1 expression was strongly proportionate to URI expression ( $p=0.026$, Fisher's exact test).

The above analysis used the median H-score to define two broad expression classes; many similar individual values (near the median) were segregated. To more precisely examine potential associations between immunohistochemistry (IHC) and ISH findings, we performed a correlation analysis using pure immunohistochemistry characteristics as staining intensity or percentage of positive cells. However, as indicated in the correlation plot (Supplementary Figure S1), the relationships between 19q12 ISH and both intensity and fraction of Cyclin E1 and URI positive cells were low.

\section{Aberrant p53 immunohistochemistry is related to $19 q 12$ amplification}

Since TP53 mutations are common in EC type II, we examined the associations of p53 immunohistochemical staining pattern with 19q12 amplification status, Cyclin E1 expression, and URI expression. According to the WHO classification 2014, aberrant p53 staining (diffuse and strong positivity in $>75 \%$ tumour cells or complete lack of staining) correlates with a TP53 mutation, while variable staining intensity in less than $75 \%$ of the tumour cells is related to wild-type TP53 (WHO classification 2014). Using this classification, we observed a significant association between aberrant TP53 expression and 19q12 amplification status (Table 3). High Cyclin E1 expression was significantly more common in EC with aberrant p53 expression ( $p=0.033$, Fisher's exact test), while URI expression was unrelated to p53 expression ( $p=0.53$, Fisher's exact test).

\section{9q12 amplification confirmation by OncoScan assay and computational methods}

For ISH assay validation, we conducted the OncoScan assay in a subset of 21 EC samples with known histological grade and ISH-determined CN. Totally, 34-214 ng dsDNA was obtained from these samples. Although Affymetrix recommends 80 ng genomic dsDNA as the input for the OncoScan FFPE Assay, all 21 samples fulfilled the quality control criteria for amplified and cleaved probes. The parameters measured from the signal intensities, such as MAPD and ndSNP quality control, were also considered acceptable for determining CN.

Consistent with the ISH assay, CNVs (measured by OncoScan) were more frequent in high-grade and nonendometrioid EC compared to low-grade endometrioid EC (Supplementary Figure S2). We observed 19q12 CN gains in 9/21 EC samples using the OncoScan FFPE Assay (Table 4), high $\mathrm{CN}$ gain $(\mathrm{CN}>3)$ in six cases (shown in Figure 1E) and $2<\mathrm{CN} \leq 3$ in three cases. Additionally to CCNE1 and URI, other 19q12 locus members, including POP4 and PLEKHF1, showed the same CN levels.

The OncoScan platform detected high 19q12 CN in all five EC samples determined as high amplification by ISH (19q12/chr19 ratio $\geq 3$; Table 4$)$. One high-grade endometrioid EC sample with apparent low 19q12 amplification by ISH (Case 27) showed high $\mathrm{CN}$ by OncoScan. Conversely, of the 12 EC samples with low amplification determined by $19 q 12$ ISH (19q12/chr19 ratio $\geq 2-3$ ), four showed $\mathrm{CN}$ gain and eight showed stable $\mathrm{CN}$ in the OncoScan assay. Of these 12 EC samples with low 19q12 amplification determined by ISH, only high-grade, non-endometrioid EC (3/6) but no low-grade endometrioid EC (0/5) samples displayed $\mathrm{CN}$ changes at $19 \mathrm{q} 12$ by the OncoScan assay. Four low-grade endometrioid EC samples showed no CNVs at $19 \mathrm{q} 12$ in either of the two assays.

We frequently observed TP53 alterations in ECs with $19 q 12 \mathrm{CN}$ gain compared to ECs without $19 \mathrm{q} 12 \mathrm{CN}$ alterations. $19 \mathrm{q} 12$ amplification/CN gain was related to TP53 loss of heterozygosity ( $\mathrm{LOH})$ and an aberrant p53 immunohistochemistry pattern suggesting mutation (but not deletion) (Table 5).

Statistical analysis revealed a high concordance between manual assessment of 19q12 ISH and OncoScanbased $\mathrm{CN}$ analysis $(\mathrm{r}=0.92$, Figure $2 \mathrm{~A})$. We further used the ISHProfiler algorithm in the same subset of 21 ECs [16]. Manually evaluated 19q12 ISH CN highly correlated with computational ISHProfiler $\mathrm{CN}$ ( $\mathrm{r}=0.89$, Figure $2 \mathrm{~B})$. A similar correlation was observed between $19 \mathrm{q} 12 \mathrm{CN}$ by OncoScan assay and by ISHProfiler $(r=0.82$, Figure $2 \mathrm{C})$. In terms of a negative control, we demonstrate a no existing relation between manual 19q12 ISH CN and TP53 $\mathrm{CN}(\mathrm{r}=-0.1012$, Figure 2D).

\section{DISCUSSION}

Current adjuvant treatment for high-grade and advanced EC is based on radiation and/or chemotherapy. However, the effectiveness of chemotherapy is uncertain and approved targeted therapies and/or predictive tests 
Table 3: Associations between 19q12 amplification and Cyclin E1, URI and p53 immunohistochemical expression levels.

\begin{tabular}{lccc}
\hline & all cases & 19q12 amplification n (\%) & p-value* \\
\hline Cyclin E1 & 144 & & 0.008 \\
low expression & 121 & $19(15.7)$ & \\
high expression & & & 0.593 \\
URI & 221 & $22(9.9)$ & \\
low expression & 45 & $6(13.3)$ & 0.026 \\
high expression & & & \\
p53 & 127 & $8(6.2)$ & \\
wild type pattern & 128 & $20(15.6)$ & \\
aberrant pattern & &
\end{tabular}

* Fisher's Exact Test

Table 4: Correspondence of 19q12 amplification status determined by in situ hybridisation (ISH) and OncoScan assay.

\begin{tabular}{|c|c|c|c|c|c|c|}
\hline \multirow[b]{2}{*}{ Case } & \multirow[b]{2}{*}{ EC subtype } & \multirow[b]{2}{*}{$\begin{array}{c}19 q 12 \text { ISH } \\
\text { amplification }\end{array}$} & \multicolumn{4}{|c|}{ 19q12 amplicon OncoScan assay } \\
\hline & & & CCNE1 & URI1 & POP4 & PLEKHF1 \\
\hline 026 & serous & high & High CN gain & High $\mathrm{CN}$ gain & High CN gain & High $\mathrm{CN}$ gain \\
\hline 033 & clear cell & high & High $\mathrm{CN}$ gain & High $\mathrm{CN}$ gain & High $\mathrm{CN}$ gain & High $\mathrm{CN}$ gain \\
\hline 042 & hg endometrioid & high & High $\mathrm{CN}$ gain & High $\mathrm{CN}$ gain & High $\mathrm{CN}$ gain & High CN gain \\
\hline 040 & serous & high & High CN gain & High $\mathrm{CN}$ gain & High $\mathrm{CN}$ gain & High $\mathrm{CN}$ gain \\
\hline 034 & hg endometrioid & high & High CN gain & High $\mathrm{CN}$ gain & High $\mathrm{CN}$ gain & High $\mathrm{CN}$ gain \\
\hline 027 & hg endometrioid & low & High $\mathrm{CN}$ gain & High CN gain & High $\mathrm{CN}$ gain & High $\mathrm{CN}$ gain \\
\hline 046 & serous & low & $\mathrm{CN}$ gain & $\mathrm{CN}$ gain & $\mathrm{CN}$ gain & $\mathrm{CN}$ gain \\
\hline 029 & carcinosarcoma & low & $\mathrm{CN}$ gain & $\mathrm{CN}$ gain & $\mathrm{CN}$ gain & $\mathrm{CN}$ gain \\
\hline 045 & serous & low & $\mathrm{CN}$ gain & $\mathrm{CN}$ gain & $\mathrm{CN}$ gain & $\mathrm{CN}$ gain \\
\hline 028 & hg endometrioid & low & - & - & - & - \\
\hline 031 & $\lg$ endometrioid & low & - & - & - & - \\
\hline 035 & $\lg$ endometrioid & low & - & - & - & - \\
\hline 036 & $\lg$ endometrioid & low & - & - & - & - \\
\hline 037 & serous & low & - & - & - & - \\
\hline 038 & hg endometrioid & low & - & - & - & - \\
\hline 039 & $\lg$ endometrioid & low & - & - & - & - \\
\hline 041 & $\lg$ endometrioid & low & - & - & - & - \\
\hline 030 & $\lg$ endometrioid & no & - & - & - & - \\
\hline 047 & $\lg$ endometrioid & no & - & - & - & - \\
\hline 048 & $\lg$ endometrioid & no & - & - & - & - \\
\hline 043 & $\lg$ endometrioid & no & - & - & - & - \\
\hline
\end{tabular}

lg: low grade; hg: high grade 
Table 5: Contribution of 19q12 amplification status to TP53 alterations.

\begin{tabular}{|c|c|c|c|c|c|c|}
\hline Case & EC subtype & $\begin{array}{c}19 q 12 \text { ISH } \\
\text { amplification }\end{array}$ & $\begin{array}{c}\text { OncoScan } \\
\text { 19q12 amplicon }\end{array}$ & $\begin{array}{c}\text { OncoScan } \\
\text { TP53 }\end{array}$ & $\begin{array}{c}\text { p53 IHC } \\
\text { positive cells }\end{array}$ & $\begin{array}{c}\text { p53 IHC } \\
\text { pattern }\end{array}$ \\
\hline 026 & serous & high & High $\mathrm{CN}$ gain & $\mathrm{LOH}$ & $0 \%$ & aberrant \\
\hline 033 & clear cell & high & High CN gain & LOH, CN loss & $95 \%$ & aberrant \\
\hline 042 & hg endometrioid & high & High CN gain & $\mathrm{CN}$ gain & $30 \%$ & wt \\
\hline 040 & serous & high & High $\mathrm{CN}$ gain & $\mathrm{LOH}$ & $100 \%$ & aberrant \\
\hline 034 & hg endometrioid & high & High $\mathrm{CN}$ gain & $\mathrm{LOH}, \mathrm{CN}$ loss & $100 \%$ & aberrant \\
\hline 027 & hg endometrioid & low & High CN gain & $\mathrm{LOH} / \mathrm{CN}$ gain & $0 \%$ & aberrant \\
\hline 046 & serous & low & $\mathrm{CN}$ gain & $\mathrm{LOH} / \mathrm{CN}$ gain & $0 \%$ & aberrant \\
\hline 029 & carcinosarcoma & low & $\mathrm{CN}$ gain & $\mathrm{LOH}$ & $100 \%$ & aberrant \\
\hline 045 & serous & low & $\mathrm{CN}$ gain & $\mathrm{LOH}$ & $100 \%$ & aberrant \\
\hline 028 & hg endometrioid & low & $\mathrm{x}$ & $\mathrm{LOH}$ & $10 \%$ & wt \\
\hline 031 & $\lg$ endometrioid & low & $\mathrm{x}$ & $\mathrm{x}$ & $0 \%$ & aberrant \\
\hline 035 & lg endometrioid & low & $\mathrm{x}$ & $\mathrm{x}$ & $30 \%$ & wt \\
\hline 036 & lg endometrioid & low & $\mathrm{x}$ & $\mathrm{x}$ & $0 \%$ & aberrrant \\
\hline 037 & serous & low & $\mathrm{x}$ & $\mathrm{LOH}$ & $100 \%$ & aberrrant \\
\hline 038 & hg endometrioid & low & $\mathrm{x}$ & $\mathrm{x}$ & $20 \%$ & $\mathrm{wt}$ \\
\hline 039 & lg endometrioid & low & $\mathrm{x}$ & $\mathrm{x}$ & $15 \%$ & wt \\
\hline 041 & lg endometrioid & low & $\mathrm{x}$ & $\mathrm{x}$ & $5 \%$ & wt \\
\hline 030 & lg endometrioid & no & $\mathrm{x}$ & $\mathrm{x}$ & $0 \%$ & aberrrant \\
\hline 047 & lg endometrioid & no & $\mathrm{x}$ & $\mathrm{x}$ & $5 \%$ & wt \\
\hline 048 & lg endometrioid & no & $\mathrm{x}$ & $\mathrm{x}$ & $5 \%$ & wt \\
\hline 043 & lg endometrioid & no & $\mathrm{x}$ & $\mathrm{x}$ & n.a. & n.a. \\
\hline
\end{tabular}

lg: low grade; hg: high grade; LOH: loss of heterozygosity

for patient selection are not available. To guide treatment decisions, detailed knowledge of oncogenic drivers is required. TCGA data has shown that solid carcinomas such as high-grade serous ovarian carcinomas and serouslike endometrial carcinomas are frequently driven by $\mathrm{CN}$ alterations rather than by somatic mutations [2, 17]. The 'copy number high' TCGA subgroup of EC is characterised by amplification of $M Y C, E R B B 2, C C N E 1$, FGFR3 and SOX17. We therefore investigated the $19 \mathrm{q} 12$ amplicon, including the oncogenic drivers CCNE1 and $U R I$, in a large EC cohort including different histological types. We observed 19q12 (CCNE1/URI) amplification in $10 \%$ of the entire EC group, most frequently in advanced stage non-endometrioid and high-grade endometrioid carcinomas (type II). This is consistent with TCGA results demonstrating significant CCNE1 (19q12) amplification in nearly $22 \%$ of the 'serous-like/cluster 4' tumours [2]. Using SNP arrays, Kuhn and colleagues recently reported CCNE1 amplification in $26.1 \%$ uterine serous carcinomas [3]. They also found amplification in up to $45 \%$
(20 out of 44) of the serous carcinomas using FISH [4]. If we consider only the high-grade and non-endometrioid ECs in our cohort, the amplified cases constitute $40.5 \%$ $(15 / 37)$.

Our recently established $19 \mathrm{q} 12$ ISH assay covers not only CCNE1 but also URI. These genes are located in close proximity, but it is unclear if $U R I$ is also an oncogenic driver of this amplicon. We and others have recently shown that $U R I(C 19$ orf 2$)$ is amplified in a subset of ovarian carcinomas and ovarian cancer cell lines $[5,6]$, suggesting that CCNE1 may not be the exclusive driver at $19 \mathrm{q} 12$. The exact role of URI in EC is largely unknown but we previously demonstrated URI amplification by FISH in $4.6 \%(5 / 108)$ of these carcinomas [6].

To validate $19 q 12$ amplification status as assessed by ISH and to explore the $19 \mathrm{q} 12$ locus, we subjected 21 ISH-characterised EC samples to OncoScan assay, which enables accurate whole genome $\mathrm{CN}$ estimation. As expected, we observed a different profile and frequency of DNA CN alterations among histological EC subtypes. 
Consistent with TCGA findings, high-grade endometrioid and serous carcinomas were characterised by extensive $\mathrm{CN}$ changes compared to low-grade endometrioid EC. This assay further confirmed our manual 19q12 ISH assessment. All carcinomas with high 19q12 amplification levels as detected by ISH $(19 \mathrm{q} 12 / \operatorname{chr} 19$ ratio $\geq 3)$ displayed high $\mathrm{CN}$ gain by OncoScan. However, a few carcinomas with low amplification as assessed by $19 q 12$ ISH (19q12/chr19 ratio $\geq 2$ but $<3$ ) showed high $\mathrm{CN}$ gain by OncoScan. Of note, only high-grade EC (4/7) with low

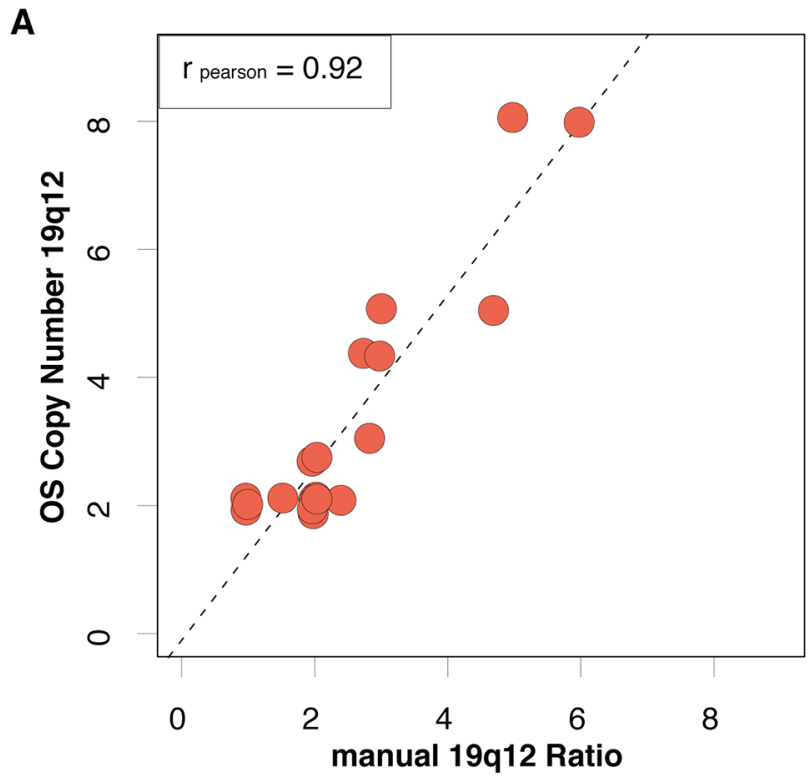

C

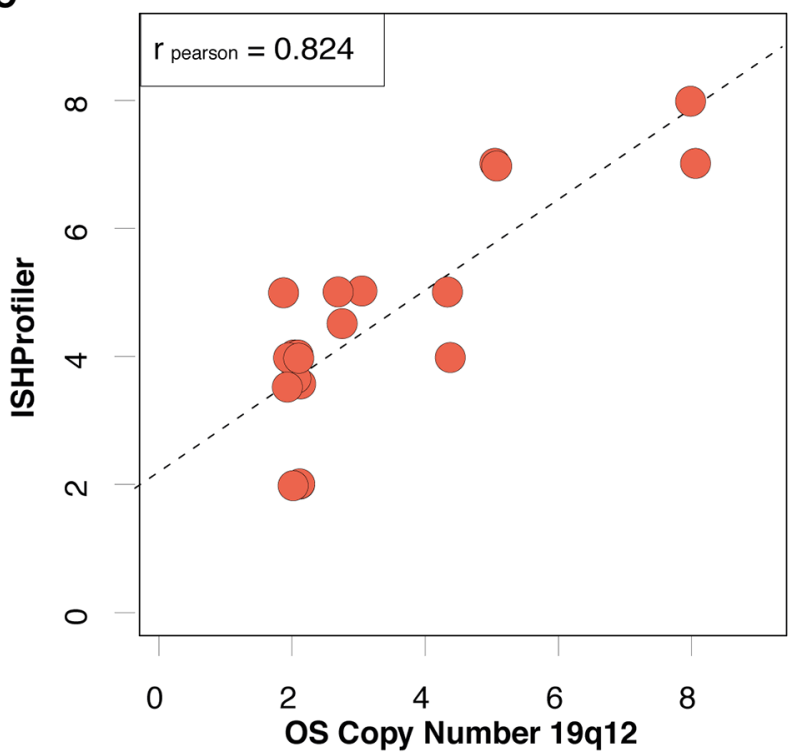

level 19q12 amplification by ISH showed CN gain by OncoScan, suggesting ISH may have lower sensitivity for $\mathrm{CN}$ gain detection, at least under certain conditions. The evaluation and stratification of 19q12 ISH was performed according to our recent study based on previously published recommendations [4, 7]. Alternatively, OncoScan technology allows for more detailed resolution and subdivision and thus may be superior for low $\mathrm{CN}$ gain. In this case, the clinical significance of $19 q 12 /$ chr19 ratio $\geq 2$ but $<3$ by ISH should be re-assessed. It must be
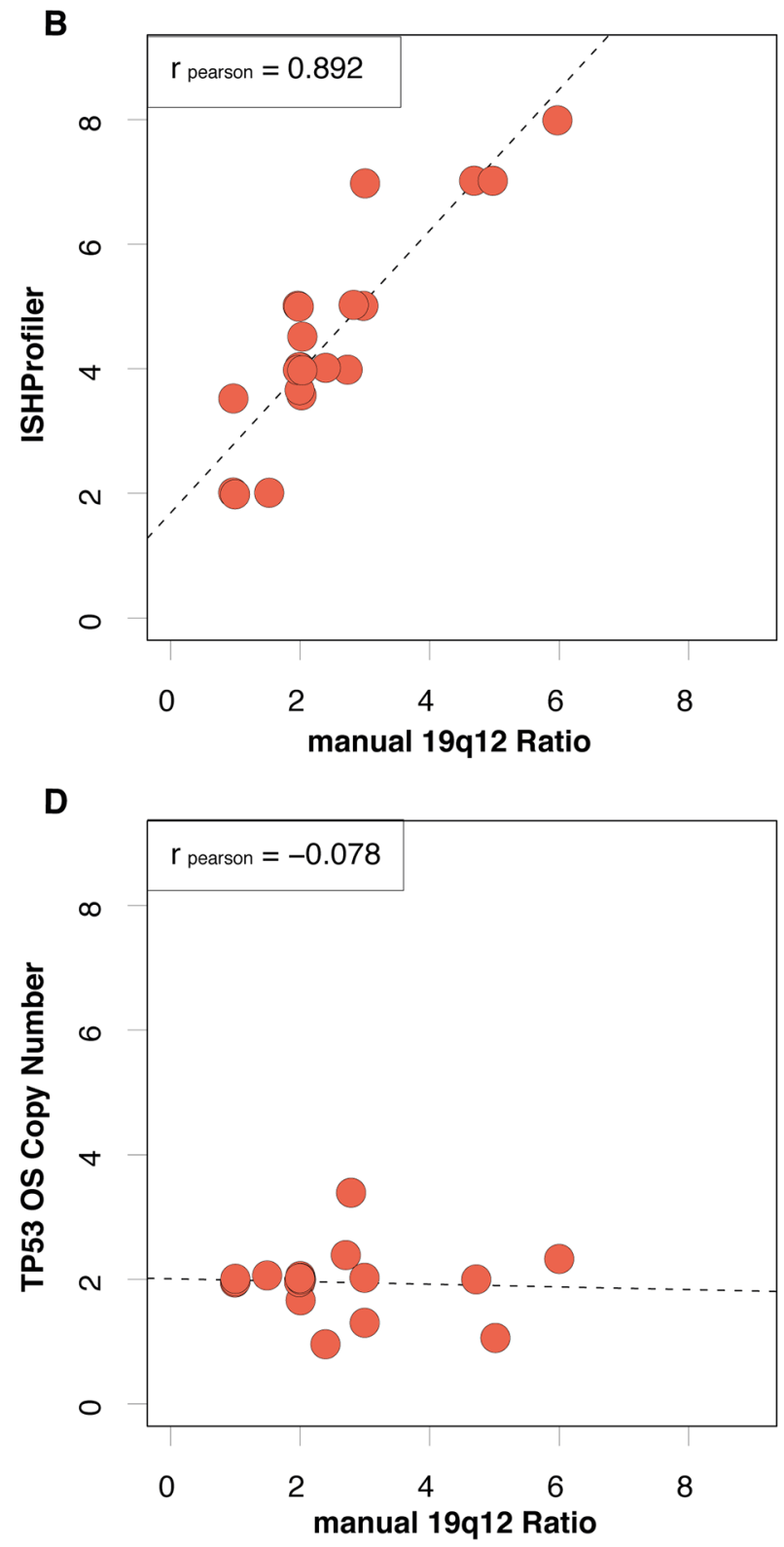

Figure 2: Correlations among 19q12 copy numbers as measured manually by ISH, OncoScan (OS) or by ISHProfiler algorithm. A. Pearson correlation of manually assessed 19q12 ISH ratio (cut-off $\geq 2$ ) and copy number at the 19q12 locus by OncoScan. Data points with exactly the same $\mathrm{x}$ - and y-values are separated by data jittering. The trend between $19 \mathrm{q} 12$ ratio and $\mathrm{CN}$ based on linear regression is plotted as a dashed line. B. The same correlation for manually assessed 19q12 ISH ratio and ISHProfiler ratio. C. The same correlation for ISHProfiler ratio and OS copy number at the 19q12 locus. D. In contrast, no correlation for the manually assessed ISH ratio and OS copy number at the TP53 locus. 
noted, however, that manual evaluation of ISH staining was conducted on tissue microarrays with limited amounts of tumour tissue. Analysis of whole tissue sections would likely yield more accurate $\mathrm{CN}$ estimates and better agreement with OncoScan.

The OncoScan assay also revealed additional genomic events at the $19 \mathrm{q} 12$ amplicon. Concurrent $\mathrm{CN}$ gains were observed not only for CCNE1 and URI but for six additional genes in close vicinity: C19Orf12, POP4, PLEKHF1, VSTM2B, UQCRFS1 and LOC284395. $C C N E 1$ was once considered the exclusive oncogenic driver within the 19q12 amplicon [18], but the present study and recent studies on ovarian and breast carcinomas have identified co-amplified genes $[6,19]$ that may also contribute to tumour aggression or treatment resistance $[6,7]$. In our recent study, 75\% URI-amplified epithelial ovarian carcinomas (EOCs) showed co-amplification of CCNE1 (nine out of 12), suggesting sole amplification of $U R I$ occurs in $25 \%$ EOC cases. On a functional level, $U R I$ but not CCNE1 enhanced the viability of ovarian cancer cell lines in a high-throughput siRNA screen [5]. In another study, CCNE1 was amplified in only five of 16 primary breast carcinomas harbouring amplification of the $19 q 12$ locus [19]. It was further demonstrated by microarray gene expression analysis that CCNE1, C19Orf2 (URI), C19Orf12, POP4, PLEKHF1 and UQCRFS1 were significantly overexpressed when amplified and that silencing of CCNE1, POP 4 and PLEKHF1 reduced cell viability. Accordingly, $C C N E 1$ is not the only driver of the 19q12 amplicon. Therefore, other 19q12 amplicon genes should be included in amplification assays.

The TCGA datasets revealed a genomic relationship between endometrial serous-like and high-grade serous ovarian carcinomas (HGSOC) [2]. Both are characterised by high CNVs and frequent TP53 mutations. Similar to high-grade and non-endometrioid EC, amplification of the 19q12 (CCNE1) locus has been reported in up to $30 \%$ $[17,20-22]$ and $U R I$ in $10 \%-15 \%$ HGSOCs $[6,7]$. In both EC and HGSOC, we found an association between 19q12 amplification (CCNE1/URI) and loss or increased p53 immunohistochemical expression, suggestive of TP53 mutation [7]. This observation is supported by the subset of EC samples subjected to OncoScan showing a relationship between $19 \mathrm{q} 12$ amplification/CN gain and TP53 LOH. Alternatively, Kuhn et al. found no significant correlation between CCNE1 gene amplification assessed by FISH and TP53 mutation in uterine serous carcinomas [4].

$19 q 12$ amplification status is a critical chemotherapy response determinant and therapeutic target in other cancer therapies. Primary treatment failure (platinumbased chemotherapy) in ovarian cancer was attributed to CCNE1 amplification [11] and partly to an intact BRCA1/2 pathway [23]. URI amplification may also mediate resistance to cisplatin in ovarian cancer cells [6]. CCNE1-amplified ovarian cancer cells were more sensitive to cell cycle arrest, growth inhibition and apoptosis induction by CCNE1 siRNA [21]. Additionally, $C C N E 1$ function can be modulated via its partner kinase CDK2 [24]. CCNE1-amplified breast cancer cells were sensitive to $C D K 2$ inhibitors, resulting in reduced cancer cell survival [19]. The relevance of 19q12 amplicon/CDK2 as a predictive marker for chemotherapy response and anti-19q12/CDK2-targeted therapies in EC remains unknown. Clinical trials are needed to evaluate whether 19q12 (CCNE1/URI) amplicon status can help identify patients most likely to respond to standard treatment or benefit from therapeutic approaches targeting cell cycle checkpoints. Our novel automated 19q12 ISH is a feasible biomarker assay and could be applied for routine diagnosis. Whether a combination of both 19q12 ISH assay and protein expression analysis of Cyclin E1 and URI by IHC is suitable, similar to HER2 assessment in breast cancer, needs further investigation.

\section{MATERIALS AND METHODS}

\section{EC sample cohort}

Primary EC samples were collected between 1985 and 2005, fixed in 4\% neutral buffered formaldehyde, embedded in paraffin and stored in the archives of the Institutes of Pathology, University of Basel and University of Zurich (Switzerland) [25]. Routine haematoxylin and eosin sections were processed for additional histopathological evaluation. Histological subtype and grade were reviewed and defined (WHO classification 2014). Tumour stage was assessed according to International Federation of Gynaecology and Obstetrics (FIGO) staging. Two tissue microarrays (TMAs) containing 436 ECs were used. Samples from the Institute of Basel were assembled into a new TMA $(n=248)$, while the Zurich-TMA was previously constructed $(\mathrm{n}=188)$ [25]. Both TMAs included two tissue cores of each tumour. Patients with localised disease underwent hysterectomy and bilateral salpingo-oophorectomy (with/without pelvic and para-aortic lymphadenectomy). High-grade and myometrium invasive EC samples received adjuvant postoperative vaginal radiation and/or chemotherapy. Clinical outcome was available in 333 cases. Median follow-up was 42 months (1-184 months). The study was approved by the Cantonal Ethics Committee of Basel and Zurich (KEK-ZH-NR: 2010-0358).

\section{9q12 amplicon detection using a dual-colour ISH automated assay}

Tissue microarrays were analysed for $19 \mathrm{q} 12$ amplification status using a DNA probe set (Ventana Medical Systems, Tucson, Arizona), by measuring the copy number ratio of the $19 \mathrm{q} 12$ amplicon to the chromosome 19. The 19q12 ISH probe (Ventana) is a 
2,4-dinitrophenyl (DNP)-labelled DNA probe, free of repetitive DNA sequences that covers a $\sim 560 \mathrm{~kb}$ span of chromosome 19q12 containing the CCNE1 and URI coding sequences. Due to homology within the alphasatellite sequences of chromosomes 1 and 5, specifically identifying chromosome 19 is difficult. Therefore, a second repeat-free DNA probe was developed to allow chromosome 19 copy number enumeration. This digoxigenin (DIG)-labelled probe hybridises to a span of $\sim 600 \mathrm{~kb}$ within $19 \mathrm{p} 13.2-19 \mathrm{p} 13.3$, including the coding sequences for the insulin receptor INSR. The dual-colour ISH assay was previously established and automated using the Ventana BenchMark XT platform [7]. Briefly, after deparaffinisation, the TMA slides were treated with cell conditioning 2 for four 12-min cycles followed by ISH protease 2 for 8 or $12 \mathrm{~min}$. After co-denaturation, 19q12 DNP and INSR DIG probes were hybridised at $51^{\circ} \mathrm{C}$ for 4 hours and washed three times at $68^{\circ} \mathrm{C}$ for 8 minutes/ wash. The $19 \mathrm{q} 12$ DNP and INSR DIG signals were detected using Ventana ultraView SISH DNP and Ventana ultraView RED ISH DIG detection kits, respectively. Tumour samples were counterstained with haematoxylin II and bluing reagent.

Before CN enumeration and further analysis, samples were evaluated for acceptable staining based on the presence of appropriate signals in normal cells and adequate signal-to-background strength. Samples that failed to meet these criteria were rejected. After identifying regions for analysis, 19q12 and INSR signal numbers within 50 representative cells were recorded. In TMA cases containing small tumour tissue amounts, the total $\mathrm{CN}$ from 50 nuclei was obtained from multiple cores. Amplification was defined as the ratio of the average number of $19 \mathrm{q} 12$ copies to the average number of INSR copies/cell. Both parameters are detectable on one slide and appear as black (19q12) and red (INSR on chr19) dots. A sample was considered amplified if $19 \mathrm{q} 12 /$ INSR ratio was $\geq 2.0[7,15]$.

\section{Cyclin E1, URI and p53 immunohistochemistry (IHC)}

TMA slides were incubated with a monoclonal antibody against Cyclin E1 (clone HE12, Santa Cruz Biotechnology, CA). Nuclear expression was scored according to the intensity of the immunostaining (0no staining; 1-weak; 2-moderate; 3-strong) and the percentage of positive tumour cells. Afterwards, the $\mathrm{H}$-score was calculated (ranging from 0-300) [7, 21]. A rabbit monoclonal antibody specific for URI (121, Ventana) was applied to TMAs and cytoplasmic expression was scored as above. The EC samples were investigated for $\mathrm{p} 53$ expression by immunohistochemistry (Dako, M7001) [26]. Tumour p53 expression strength was scored according to p53-positive cell numbers: $0 \%, 1 \%-$ $75 \%$ or $>75 \%$ (WHO Classification of Tumours of Female Reproductive Organs 2014).

\section{OncoScan CN assay}

To independently confirm CN results from $19 \mathrm{q} 12$ ISH, we performed the OncoScan assay (Affymetrix, Inc) based on Molecular Inversion Probe (MIP) technology to analyse whole-genome $\mathrm{CN}$ variations (CNVs) in a 21 EC sample subset. The 19q12 ISH amplification status ranged from high $\mathrm{CN}$ in five $\mathrm{EC}$ samples $(19 \mathrm{q} 12 / \mathrm{Chr} 19$ ratio $>3)$ to low amplification in $12(19 \mathrm{q} 12 / \mathrm{Chr} 19$ ratio $2-3)$ and no amplification in four $(19 \mathrm{q} 12 / \mathrm{Chr} 19$ ratio $<2)$. For the OncoScan assay, formalin-fixed and paraffin-embedded (FFPE) cancer tissue blocks from 1985-1995 were punched and the removed sections $(3-5$ cylinders, $0.6-\mathrm{mm}$ diameter) deparaffinised with xylene. After ethanol washing, genomic DNA was extracted with the DNeasy Blood and Tissue Kit (Qiagen \#69504) following the manufacturer's protocol. The double-strand DNA concentration (dsDNA) was determined using the fluorescence-based Qubit dsDNA HS Assay Kit. The samples were further processed by IMGM Laboratories GmbH (Martinsried, Germany) for CNV determination according to the Affymetrix OncoScan FFPE assay recommended protocol.

Briefly, the assay uses locus-specific molecular inversion probes (MIPs) optimised for highly degraded FFPE samples with a 40-bp probe interrogation site. MIPs were hybridised to complementary DNA fragments leaving a gap at the SNP position of interest. The MIPs were circularised by adding a gap-filling enzyme, ligase and nucleotide complementary to the locus being interrogated. The gap-filling reaction was performed in separate steps for $\mathrm{A} / \mathrm{T}$ and $\mathrm{G} / \mathrm{C}$ nucleotides to properly distinguish between signals. After linear DNA fragment digestion (including gDNA and nonfilled probes), the circularised probes were cleaved by HaeIII and amplified by PCR using common primers. The resulting probes (50 and $70 \mathrm{bp}$ ) were biotinylated and hybridised to separate OncoScan microarrays for $\mathrm{A} / \mathrm{T}$ and $\mathrm{G} / \mathrm{C}$ signals/sample. Signal strength gives information on the initial $\mathrm{CN}$ in a region of interest; SNPs help distinguish alleles. After hybridisation, microarrays were scanned with a 3000-7G GeneChip scanner (Affymetrix) and image files processed to yield signal intensity (.cel-) files. Cel files were then converted to .OSCHP files using OncoScan Console 1.3 (Affymetrix, Inc.), yielding normalised log intensity ratios (sample/reference), B-allele frequencies and a set of metrics for quality control, including a median of absolute pair-wise difference (MAPD), normal diploid SNP QC (ndSNPQC) or normal diploid waviness standard deviation (ndWavinessSD). After quality checking, samples were categorised as high, normal or low $\mathrm{CN}$ based on the log intensity ratio using the TuScan algorithm as implemented in Nexus Express Software for OncoScan 3.0.1 (Biodiscovery, Inc. 2014, CA, USA). 


\section{Image digitisation}

The bright field and fluorescence slide scanner Axio Scan.Z1 (Carl Zeiss, Jena, Germany) was used to digitise tissue cores at a resolution of $\times 40(0.11 \mu \mathrm{m} /$ pixel $)$, according to the manufacturer's instructions.

\section{Image-based computational workflow (ISHProfiler)}

Tissue cores were digitised and pre-processed (white balancing, deconvolution and contrast modification) using the scanner's default auto-correction settings. Images were then resized by bicubic interpolation to $4096 \times 4096$ pixels for efficient tiling $(4096=212)$. These modified images served as input data for the computational workflow ISHProfiler. The computational workflow was implemented in MATLAB (R2014b) and tested on MacPro (2014). MATLAB built-in functions for the circular Hough transform (imfindcircles) and ROC analysis (perfcurve) were used. The software package LIBSVM (version 3.18) was used to train, validate and test support vector machine (SVM) models on the data.

\section{Statistics}

Statistical analyses were conducted using IBM SPSS software (version 22). Associations between 19q12 amplification status and pathological parameters and Cyclin E1, URI and p53 expression levels were assessed by Pearson's chi-square or Fisher's exact test (twosided). Differences in overall survival were determined by Kaplan-Meier analysis, and statistical significance was calculated using the log-rank test. Univariate and multivariate survival analyses were performed using the COX model of proportional hazards. $P \leq 0.05$ was considered statistically significant. Pearson correlation analysis was conducted using R [R Core Team (2013). $\mathrm{R}$ : A language and environment for statistical computing. R Foundation for Statistical Computing, Vienna, Austria. ISBN 3-900051-07-0, URL https://www.r-project.org.

\section{ACKNOWLEDGMENTS}

We would like to thank Martina Storz, André Fitsche, Annette Bohnert, Susanne Dettwiler, Mathias Drach, Alisa Tubbs, and Leigh-Ann Henricksen for technical and study coordination assistance. Supported by grants from Oncosuisse (Krebsliga Schweiz) to Peter Wild.

\section{CONFLICTS OF INTEREST}

H.M. and A.N. have received research funds from Roche Ventana Medical Systems, Inc. to perform in situ hybridisation assays free of charge. Roche Ventana played no role in the design and conduct of the study or in the collection, analysis or interpretation of the data and has had no involvement in the preparation, review or approval of the manuscript. No conflict of interest exists for all other authors.

\section{REFERENCES}

1. Bokhman JV. Two pathogenetic types of endometrial carcinoma. Gynecol Oncol. 1983; 15:10-17.

2. Cancer Genome Atlas Research Network, Kandoth C, Schultz N, Cherniack AD, Akbani R, Liu Y, Shen H, Robertson AG, Pashtan I, Shen R, Benz CC, Yau C, Laird PW, Ding L, et al. Integrated genomic characterization of endometrial carcinoma. Nature. 2013; 497:67-73.

3. Kuhn E, Wu RC, Guan B, Wu G, Zhang J, Wang Y, Song L, Yuan X, Wei L, Roden RB, Kuo KT, Nakayama K, Clarke $\mathrm{B}$, et al. Identification of molecular pathway aberrations in uterine serous carcinoma by genome-wide analyses. Journal of the National Cancer Institute. 2012; 104:1503-1513.

4. Kuhn E, Bahadirli-Talbott A and Shih Ie M. Frequent CCNE1 amplification in endometrial intraepithelial carcinoma and uterine serous carcinoma. Mod Pathol. 2014; 27:1014-1019.

5. Davis SJ, Sheppard KE, Pearson RB, Campbell IG, Gorringe KL and Simpson KJ. Functional analysis of genes in regions commonly amplified in high-grade serous and endometrioid ovarian cancer. Clin Cancer Res. 2013; 19:1411-1421.

6. Theurillat JP, Metzler SC, Henzi N, Djouder N, Helbling M, Zimmermann AK, Jacob F, Soltermann A, Caduff R, Heinzelmann-Schwarz V, Moch H and Krek W. URI is an oncogene amplified in ovarian cancer cells and is required for their survival. Cancer Cell. 2011; 19:317-332.

7. Noske A, Henricksen LA, LaFleur B, Zimmermann A, Tubbs A, Singh S, Storz M, Fink D and Moch H. Characterization of the $19 \mathrm{q} 12$ amplification including CCNE1 and URI in different epithelial ovarian cancer subtypes. Experimental and molecular pathology. 2014; 98:47-54.

8. Gstaiger M, Luke B, Hess D, Oakeley EJ, Wirbelauer C, Blondel M, Vigneron $M$, Peter $M$ and Krek W. Control of nutrient-sensitive transcription programs by the unconventional prefoldin URI. Science. 2003; 302:1208-1212.

9. Galaal K, Al Moundhri M, Bryant A, Lopes AD and Lawrie TA. Adjuvant chemotherapy for advanced endometrial cancer. Cochrane Database Syst Rev. 2014; 5:CD010681.

10. Moxley KM and McMeekin DS. Endometrial carcinoma: a review of chemotherapy, drug resistance, and the search for new agents. Oncologist. 2010; 15:1026-1033.

11. Etemadmoghadam D, deFazio A, Beroukhim R, Mermel C, George J, Getz G, Tothill R, Okamoto A, Raeder MB, Harnett P, Lade S, Akslen LA, Tinker AV, et al. Integrated genomewide DNA copy number and expression analysis identifies 
distinct mechanisms of primary chemoresistance in ovarian carcinomas. Clin Cancer Res. 2009; 15:1417-1427.

12. Patch AM, Christie EL, Etemadmoghadam D, Garsed DW, George J, Fereday S, Nones K, Cowin P, Alsop K, Bailey PJ, Kassahn KS, Newell F, Quinn MC, et al. Whole-genome characterization of chemoresistant ovarian cancer. Nature. 2015; 521:489-494.

13. Lapenna S and Giordano A. Cell cycle kinases as therapeutic targets for cancer. Nat Rev Drug Discov. 2009; 8:547-566.

14. Cicenas J and Valius M. The CDK inhibitors in cancer research and therapy. J Cancer Res Clin Oncol. 2011; 137:1409-1418.

15. Kuhn E, Bahadirli-Talbott A and Shih IM. Frequent CCNE1 amplification in endometrial intraepithelial carcinoma and uterine serous carcinoma. Mod Pathol. 2013; 27:1014-1019.

16. Zhong Q, Ruschoff JH, Guo T, Gabrani M, Schuffler PJ, Rechsteiner M, Liu Y, Fuchs TJ, Rupp NJ, Fankhauser C, Buhmann JM, Perner S, Poyet C, et al. Image-based computational quantification and visualization of genetic alterations and tumour heterogeneity. Sci Rep. 2016; 6:24146.

17. Cancer Genome Atlas Research Network. Integrated genomic analyses of ovarian carcinoma. Nature. 2011; 474:609-615.

18. Etemadmoghadam D, George J, Cowin PA, Cullinane C, Kansara M, Australian Ovarian Cancer Study Group, Gorringe KL, Smyth GK and Bowtell DD. Amplicondependent CCNE1 expression is critical for clonogenic survival after cisplatin treatment and is correlated with 20q11 gain in ovarian cancer. PLoS One. 2010; 5:e15498.

19. Natrajan R, Mackay A, Wilkerson PM, Lambros MB, Wetterskog D, Arnedos M, Shiu KK, Geyer FC, Langerod A, Kreike B, Reyal F, Horlings HM, van de Vijver MJ, et al. Functional characterization of the 19q12 amplicon in grade III breast cancers. Breast Cancer Res. 2012; 14:R53.

20. Karst AM, Jones PM, Vena N, Ligon AH, Liu JF, Hirsch MS, Etemadmoghadam D, Bowtell DD and Drapkin R. Cyclin e1 deregulation occurs early in secretory cell transformation to promote formation of fallopian tubederived high-grade serous ovarian cancers. Cancer Res. 2014; 74:1141-1152.

21. Nakayama N, Nakayama K, Shamima Y, Ishikawa M, Katagiri A, Iida K and Miyazaki K. Gene amplification CCNE1 is related to poor survival and potential therapeutic target in ovarian cancer. Cancer. 2010; 116:2621-2634.

22. Pils D, Bachmayr-Heyda A, Auer K, Svoboda M, Auner V, Hager G, Obermayr E, Reiner A, Reinthaller A, Speiser P, Braicu I, Sehouli J, Lambrechts S, et al. Cyclin E1 (CCNE1) as independent positive prognostic factor in advanced stage serous ovarian cancer patients - a study of the OVCAD consortium. Eur J Cancer. 2014; 50:99-110.

23. Etemadmoghadam D, Weir BA, Au-Yeung G, Alsop K, Mitchell G, George J, Australian Ovarian Cancer Study Group, Davis S, D’Andrea AD, Simpson K, Hahn WC and Bowtell DD. Synthetic lethality between CCNE1 amplification and loss of BRCA1. Proc Natl Acad Sci U S A. $2013 ; 110: 19489-19494$.

24. Etemadmoghadam D, Au-Yeung G, Wall M, Mitchell C, Kansara M, Loehrer E, Batzios C, George J, Ftouni S, Weir BA, Carter S, Gresshoff I, Mileshkin L, et al. Resistance to CDK2 inhibitors is associated with selection of polyploid cells in CCNE1-amplified ovarian cancer. Clin Cancer Res. 2013; 19:5960-5971.

25. Ikenberg K, Valtcheva N, Brandt S, Zhong Q, Wong CE, Noske A, Rechsteiner M, Rueschoff JH, Caduff R, Dellas A, Obermann E, Fink D, Fuchs T, et al. KPNA2 is overexpressed in human and mouse endometrial cancers and promotes cellular proliferation. The Journal of pathology. 2014; 234:239-252.

26. Wild PJ, Ikenberg K, Fuchs TJ, Rechsteiner M, Georgiev S, Fankhauser N, Noske A, Roessle M, Caduff R, Dellas A, Fink D, Moch H, Krek W, et al. p53 suppresses type II endometrial carcinomas in mice and governs endometrial tumour aggressiveness in humans. EMBO Mol Med. 2012; 4:808-824. 